\title{
Long Distance Pollen Flow in Mandarin Orchards Determined by AFLP Markers-Implications for Seedless Mandarin Production
}

\author{
Chih-Cheng T. Chao', Jinggui Fang', and Pachanoor S. Devanand² \\ Department of Botany and Plant Sciences, University of California-Riverside, Riverside, \\ CA 92521-0124 \\ AdDitional INDEX WORDS. compatibility, cross-pollination, fruit size, maximum seed content, outcrossing, pollen dispersal \\ distance, selfing
}

\begin{abstract}
Production of seedless mandarins such as 'Nules' clementine mandarin (Citrus clementina Hort. Ex Tan.) and 'Afourer' mandarin [C. sinensis $(\mathrm{L}$.) Osbeck $\times C$. reticulata Blanco] is increasing in California as consumers' interest in seedless, easy peeling, and good tasting mandarins increases. The fruit would produce seeds if cross-pollination with compatible pollen source occurred. It is almost impossible to prevent cross-pollination between compatible mandarin cultivars by honeybees (Apis mellifera L.) within the multi-faceted agricultural environment in California. To produce seedless mandarin, growers either plant a single cultivar in a large solid block or try to use pollen-sterile navel oranges (C. sinensis) or satsuma mandarins ( $C$. unshiu Marco.) as buffers to prevent cross-pollination. The question of how many rows of buffer trees or spacing can effectively prevent cross-pollination by honeybees between compatible mandarins is unclear. We initiated a study using fluorescent-labeled AFLP markers to determine the pollen parentages of 'Nules' clementine seedlings and 'Afourer' mandarin seedlings from two orchards in California. The longest distance of pollen flow at an orchard near Madera was $521 \mathrm{~m}$. The pollen of 'Minneola' tangelo (C. reticulata $\times$ C. paradisi Macf.) was able to disperse across a minimum of 92 rows of 'Lane Late' navel oranges plus two rows of 'Afourer' mandarins to pollinate 'Afourer' mandarins. We also found that all the seedlings of 'Nules' clementine mandarin at an orchard near Bakersfield had been pollinated by 'Afourer' mandarin pollen. The pollen of 'Afourer' mandarin was able to disperse up to distances between 837 and $960 \mathrm{~m}$ to pollinate 'Nules' clementine. The pollen dispersal distance found in this study was at least 16 times longer than previously reported in a citrus orchard. Growers need to consider a much larger space or buffer rows to prevent cross-pollination and produce seedless mandarins in California.
\end{abstract}

The California citrus industry is planting more mandarins as consumer and market preferences have shifted to seedless, easy peeling, and good tasting mandarins. The increased importation of clementine mandarin from Spain, Morocco, and other countries also encourages California citrus growers to plant new mandarins. Large plantings of two types of mandarins have become common in recent years. The first type is the clementine mandarin originating from Spain, Morocco, or other countries. Currently there are 16 clementine cultivars, and 'Nules' clementine (also called 'Clemenules' or 'De Nules') has the largest planting in California. Other clementine cultivars such as 'Caffin', 'Fina Sodea', and 'Sidi Aissa' also are grown at a limited scale. The second type of mandarin that is increasing in California is the 'W. Murcott Afourer' mandarin [also called 'Afourer' or 'W. Murcott' mandarin (it is a tangor, however, the exact parentage is unknown)]. Both types of mandarin have good rind color, are easy peeling, great tasting, and almost completely seedless if grown in isolation because of a self-incompatibility system (Oppenheimer, 1948; Soost, 1956, 1969). However, both groups of mandarins can produce seeds if there are compatible pollen sources nearby. Valencia orange (C. sinensis), 'Minneola'tangelo,

Received for publication 1 June 2004. Accepted for publication 8 Oct. 2004. We acknowledge funding support from the California Citrus Research Board 5100-123. We thank Horizon Farms and Paramount Citrus Inc. for assistance in this study and J. Dotty and C. Ratnayake for their technical assistance. We thank Drs. J.J. Chen, N.C. Ellstrand, and T. Kahn for critical review of the manuscript

${ }^{1}$ Assistant Cooperative Extension Specialist and Assistant Horticulturist. Corresponding author, email: ctchao@citrus.ucr.edu; phone: 951-827-3441; fax: 951-827-4437.

${ }^{2}$ Postdoctoral Researchers. and other mandarin hybrids can be excellent pollen sources for seed production in clementine mandarins (de Lange and Vincent, 1979). 'Minneola' tangelo, pummelo [C. grandis (L.) Osbeck], lemon [C. limon (L.) Burm. f.], grapefruit (C.paradisi), and some Valencia oranges have pollen germination rates higher than $35 \%$ and can potentially cause seeds in clementine mandarins (Barry, 1995). In a previous hand cross-pollination study in California, we found that 'Afourer' mandarin is a very strong pollinizer that can cause up to 43 seeds per fruit in clementine mandarins (Chao, 2005). When clementine mandarins were used as pollinizers, they could cause an average of 5-12 seeds per fruit in 'Afourer' mandarin. However, how many seed could these two groups of mandarins produce if they are planted nearby in an orchard environment was unknown.

California produces hundreds of crops, many requiring cross-pollination by honeybees to set fruit and produce seeds. It is almost impossible to exclude honeybee activity during the blooming period of mandarins in California. As the production acreages of mandarin increases, the markets demand and pay higher price for the "completely seedless" fruit. Currently, the price between "completely seedless" 'Afourer' mandarin fruit and 'Afourer' mandarin fruit with seeds could be three to four times as much, for example, $\$ 2.2 / \mathrm{kg}$ for "completely seedless" fruit vs. $\$ 0.6-0.7 / \mathrm{kg}$ for fruit with seeds (C.T. Chao, unpublished). Only few growers can afford large plantings (hundreds to thousands of hectares) of clementine mandarins or 'Afourer' mandarin in solid blocks to provide enough isolation to prevent cross-pollination by honeybees as necessary to produce seedless mandarins. Other methods that have been tried to prevent seeds in mandarins include careful site selection before planting, use 
of buffer rows or spacing, or exclusion of bee hives in the orchards and surrounding area if possible. Growers can survey the potential planting sites carefully, and determine if the distance is large enough between the compatible pollen sources and planting sites to prevent cross-pollination. Some growers also plant navel oranges or satsuma mandarins as buffers between two compatible mandarins such as 'Nules' clementine and 'Afourer' mandarin. Both navel oranges and satsuma mandarin do not produce viable pollen and, therefore, in principle, could be used as buffer trees to prevent cross-pollination by honeybees (Barry, 1995). In a previous study in Australia, Wallace et al. (2002) found the pollen of 'Ellendale' mandarin was able to transfer across more than 10 rows of trees at a distance $>50 \mathrm{~m}$. Exactly how many rows of buffer trees or space are needed to prevent cross-pollination by honeybees effectively in California is unknown.

Amplified fragment length polymorphism (AFLP) is a polymerase chain reaction based molecular marker system (Mueller and Wolfenbarger, 1999) that has been used extensively in analyses of genetic relationships of many plants in recent years. It is in general a dominant marker system, and can generate a large number of polymorphic bands with each primer set. We chose the AFLP marker system to study the issue of cross-pollination among mandarins in orchards, and to determine the pollen parentages of mandarin seedlings from fruit samples collected in mandarin orchards in California. The objectives of the study were to determine how far and across how many rows of buffer trees could pollen disperse in two orchards to cause seeds in compatible mandarins in California.

\section{Materials and Methods}

OrChard ARrangement. Fruit samples for the study were collected from two orchards in California. One orchard was located about $15 \mathrm{~km}$ north of Bakersfield, Calif., and the second site was located about $20 \mathrm{~km}$ northeast of Madera, Calif. The Bakersfield orchard had 'Afourer' mandarins on 'Carrizo' citrange rootstock [C. sinensis $\times$ Poncirus trifoliata (L.) Raf.] that was planted on the north side of the orchard in 2000, 2001, or 2002, and on the east side of the orchard in 2000. 'Nules' clementine, also on 'Carrizo' rootstock, was planted in the southwest corner of the orchard in 2000. 'Powell', 'Autumn Gold', 'Chislett', and 'Barnfield' late-season navel oranges were planted northeast of the 'Nules' clementine in 2001. Empty ground was between 'Nules' clementine and 'Afourer' mandarin to the east of the orchard (Fig. 1). There were no trees in the field between the east 'Afourer' mandarin and 'Nules' clementine in Spring 2002 (the bloom period for 2002 fruit set), but 'Barnfield' navel oranges were planted in late Spring 2002 after the bloom. The orchard was surrounded by almond [Prunus dulcis (Mill.) D.A. Webb], grape (Vitis vinifera L.), pistachio (Pistacia vera L.), and a navel orange orchard to the south. There was no other citrus nearby, except an orchard of Valencia orange located $1.1 \mathrm{~km}$ south of the orchard. The orientation of the 'Nules' clementine and 'Afourer' mandarin blocks was north-south, and the navel orange block was east-west. The planting spacing was $5.8 \times 3.1 \mathrm{~m}$ in 'Nules' block and $5.5 \mathrm{~m}$ or $6.1 \mathrm{~m}$ of row spacing in other blocks.

The field in Madera County consisted of six types of citrus (Fig. 2). All trees were on trifoliate orange rootstock (P. trifoliata) and planted in 1999 . There were 4.1 ha each of clementine mandarin cultivars Caffin (30 rows) and Fina Sodea (22 rows) at the west end of the orchard, followed by 8.1 ha of 'Owari' satsuma mandarin (30 rows), 8.1 ha of 'Afourer' mandarins (27 rows),
40.5 ha of 'Lane Late' navel oranges (92 rows), and 16.2 ha of 'Minneola' tangelos (37 rows) to the east. The orientation of the orchard is north-south with $5.5 \times 2.4 \mathrm{~m}$ planting space.

The clementine mandarins and 'Afourer' mandarin are crosscompatible and can produce seeds in each other. The navel oranges and satsuma mandarins do not produce viable pollen and were used as buffer rows to prevent pollination between clementine mandarins, 'Afourer' mandarin, and 'Minneola' tangelo. The orchard was surround by almond and rangeland and there was no other citrus nearby.

SAMPle COllection ANd SEed Germination. Fruit samples of 'Nules' clementine were collected in Nov. 2002 from the Bakersfield orchard. Fifteen fruit were collected from the 10th row at the southeast corner of the 'Nules' clementine block along a 25-tree-interval transect from north to south and from the 10th tree of the southeast end of the 'Nules' block at a 15-row interval transect from east to west. We collected the fruit samples from north to south at the eastern end of the 'Nules' block and from west to east at the southern end of the 'Nules' block. Based on presampling in the orchard, we anticipated these transect sampling should identify cross-pollination events. If the intended sampling trees had $<15$ fruit, then the neighboring trees would be sampled. Diameter and weight of all fruit samples were measured. Fruit samples of 'Afourer' mandarins were collected from the Madera site in Feb. 2003. Fifteen fruit samples of tree 10, 30, 50, 70, 90, 110,130 , and 150 from the third row from the west end and from the third row from the east end of the 'Afourer' mandarin block were collected. Based on the previous information about pollen dispersal in citrus orchard, we only sampled the east and west end of the 'Afourer' mandarin block. We expected only the trees at the edge of the block would be cross-pollinated. Diameter and weight of all fruit samples were measured. Seeds were extracted from the fruit and counted. Seedcoats were removed from the seeds and seeds were germinated on moist sterilized filter paper in petri dishes under room light for 1 week. After 1 week, germinated seeds were transferred to Jiffy-7 peat pellets (Jiffy Products Ltd., Shippagan, N.B., Canada) and grown on a Flora-Cart (Grower Supply Co., Dexter, Mich.) in the laboratory without lighting for 1 week, then under $24 \mathrm{~h}$ fluorescent light. Seedlings were grown for 2 months before true leaves were taken for DNA extraction as needed for parentage identification. Leaf samples were from 'Nules' clementine, 'Afourer' mandarin, navel oranges from the Bakersfield orchard, and Valencia orange to the south of the Bakersfield orchard. Leaf samples were also collected from 'Caffin', 'Fina Sodea' clementines, 'Owari' satsuma mandarin, 'Afourer' mandarin, 'Lane Late' navel orange, and 'Minneola' tangelo from the Madera orchard.

DNA ISOLATION AND FLUORESCENT-AFLP ANALYSIS. Total DNA was extracted from leaves using the Dneasy system (Qiagen, Valencia, Calif.). DNA concentrations were quantified using a Hoefer DyNA Quant 200 (Pharmacia Biotech, Piscataway, N.J.). AFLP analysis was conducted using the GIBCO BRLAFLP system II (Life Technologies, Grand Island, N.Y.) and the products were visualized with a LI-COR 4000-L automated sequencer (LI-COR, Lincoln, Nebr.). The AFLP protocol is described in detail in a previous study (Chen et al., 2004). Thirty-two EcoR $\mathrm{I}+2$ bases/Mse $\mathrm{I}+3$ bases AFLP primer sets $\left(\mathrm{E}+_{-} / \mathrm{M}+_{-}{ }_{-}\right)$ were initially screened for markers that were specifically linked to 'Caffin' and 'Fina Sodea' clementine mandarins from Madera, 'Owari' satsuma mandarin from Madera, 'Lane Late' navel orange from Madera, and 'Minneola' tangelo from Madera, 'Afourer' mandarin from Madera and from Bakersfield, navel oranges from 


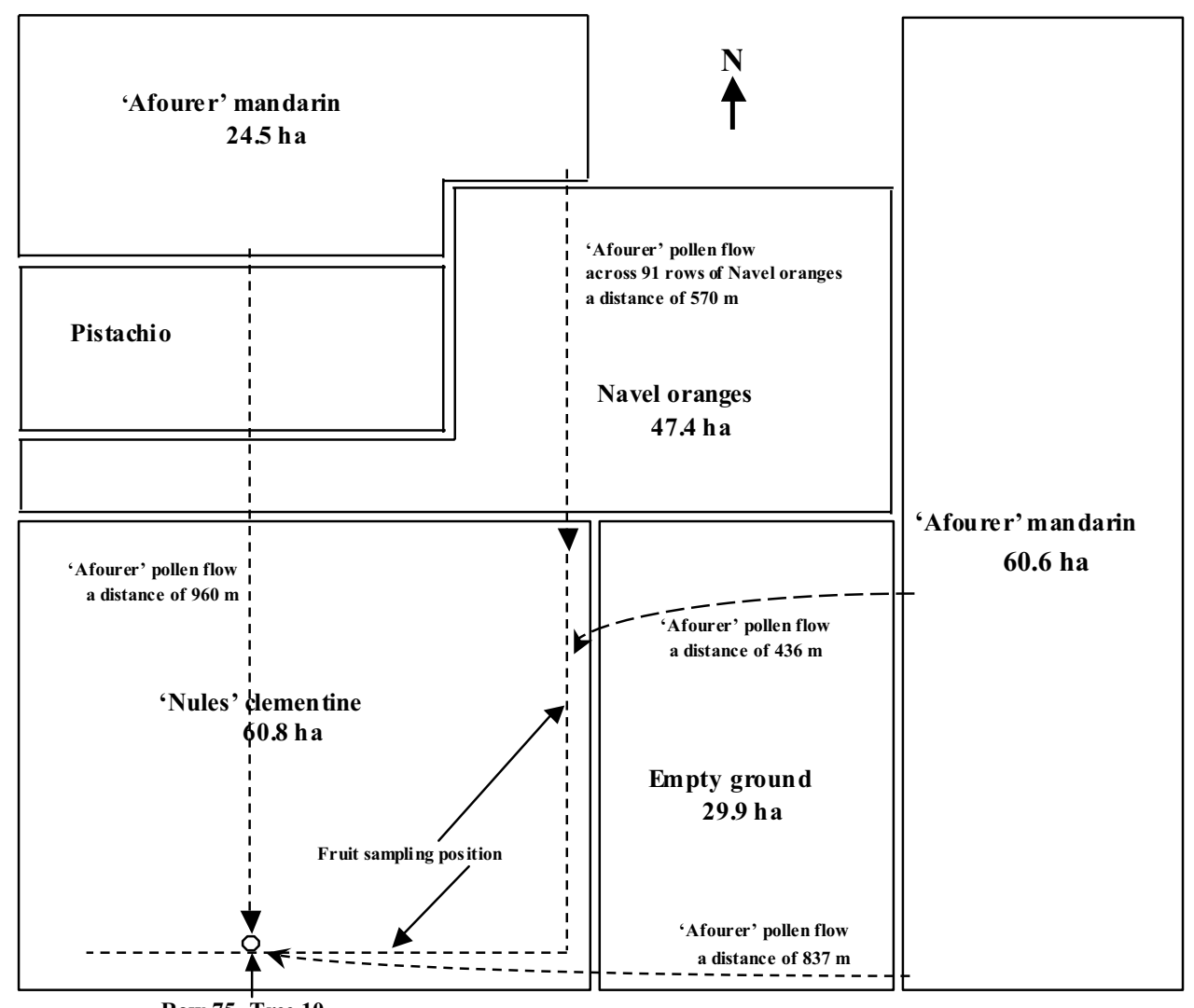

Row 75, Tree 10

Fig. 1. The name, size, and number of rows in each cultivar block, fruit sampling position within the 'Nules' mandarin block (as shown by dashed lines and indicated by arrows with solid lines), the 'Afourer' mandarin pollen dispersal direction (as shown by arrows with dashed lines), percentage of the seedlings from outcrosses (showed on top of the arrows with dashed lines), and distance of the 'Afourer' mandarins pollen transferred within an orchard near Bakersfield, Calif., found in the pollen parentage study of 'Nules' clementine seedlings.

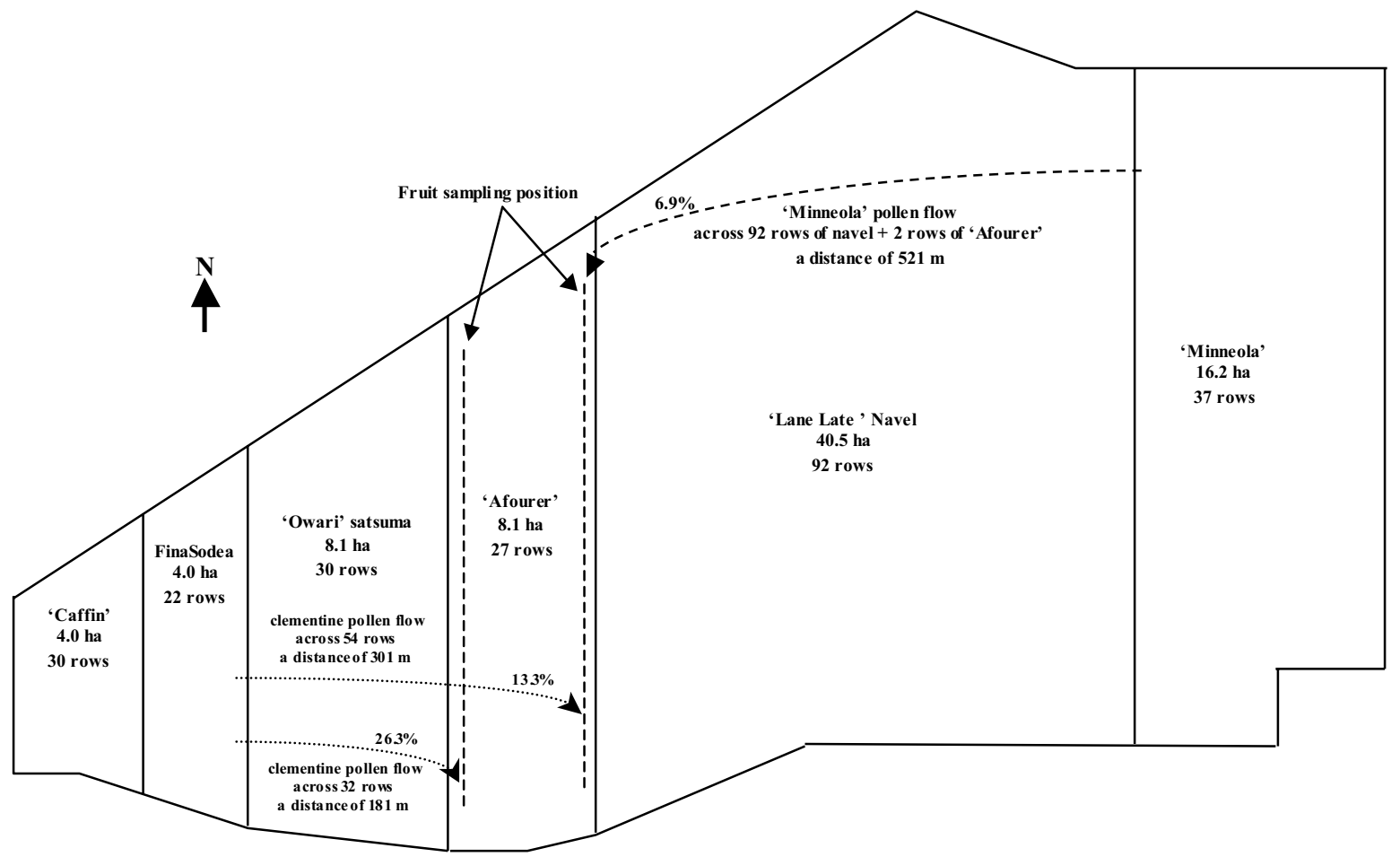

Fig. 2. The name, size, and number of row in each cultivar block, fruit sampling position within the 'Afourer' mandarin block (as shown by dashed lines and indicated by arrows with solid lines), the pollen dispersal direction (as shown by arrows with dashed lines), percentage of the seedlings from outcrosses (showed on top of the arrows with dashed lines), and the distance of the 'Minneola' tangelo or clementine mandarin pollen transferred within an orchard near Madera, Calif., that found in the pollen parentage study of 'Afourer' mandarin seedlings. 
Bakersfield, Valencia orange from south of Bakersfield orchard, and 'Nules' clementine from Bakersfield. The polymerase chain reaction (PCR) was performed using a MJR Cycle LR (MJ Research, Watertown, Mass.). AFLP bands that were present only in one cultivar but absent in all other citrus were selected as cultivar-specific AFLP markers and used to study the pollen progenitors of the seedlings.

\section{Results}

Fruit Size, fruit Weight, AND SEED NUMBER OF SAMPLes. We found seeds in all fruit samples of 'Nules' clementine from all the trees at the Bakersfield orchard. Some fruit out of 15 fruit had no seed and the average seed number per tree (from 15 fruit) ranged from 0.7 to 15.1 (Table 1). The maximum seed content per fruit ranged from 5 to 40 seeds among all sampled trees. Sampling from west to east of the 'Nules' block showed that row 60 had the highest number of seed per fruit and the seed content decreased when moving away from row 60 in both directions. Sampling from north to south showed the highest content of seed per fruit was in tree 150 and the number of seed per fruit tapered off in both directions when moving away from tree 150 . Fruit with larger numbers of seeds usually have large fruit size and heavier fruit weight (Chao, 2005).

CULTIVAR-SPECIFIC AFLP MARKERs. Thirty-two $E c o R I+2$ bases / Mse I +3 bases AFLP primer sets were screened for cultivarspecific markers. There was no difference in any banding pattern among the three clementine mandarins, the 'Nules', 'Fina Sodea', and 'Caffin'. Similar results have been reported before showing that most clementine mandarins are closely related and it is difficult to differentiate them by AFLP markers or other markers (Bretó et al., 2001). There were no differences in AFLP polymorphism among different navel oranges and Valencia oranges in the primer screening. An example of the 'Afourer' mandarin and 'Nules' clementine-specific markers, and segregation among 38 'Nules' clementine seedlings from orchard near Bakersfield

Table 1. Row and tree number of sampling sites within the 'Nules' Clementine mandarin block, number of fruit without seed (out of 15 fruit per tree), average number of seeds per tree, and maximum seed number per fruit found in the orchard near Bakersfield, Calif.

\begin{tabular}{lcccc}
\hline Row no. & Tree no. & $\begin{array}{c}\text { Fruit without } \\
\text { seeds (no.) }\end{array}$ & $\begin{array}{c}\text { Seeds/tree } \\
\text { (mean no. } \pm \text { SD) }\end{array}$ & $\begin{array}{c}\text { Max. seeds/ } \\
\text { fruit (no.) }\end{array}$ \\
\hline 15 & 10 & 5 & $4.1 \pm 6.7$ & 27 \\
30 & 10 & 10 & $1.1 \pm 2.1$ & 7 \\
45 & 10 & 7 & $2.5 \pm 4.1$ & 14 \\
60 & 11 & 0 & $15.1 \pm 12.2$ & 40 \\
75 & 10 & 8 & $3.9 \pm 9.0$ & 34 \\
90 & 10 & 6 & $2.5 \pm 5.0$ & 20 \\
105 & 10 & 10 & $1.9 \pm 4.0$ & 15 \\
120 & 11 & 9 & $0.7 \pm 1.3$ & 5 \\
135 & 11 & 8 & $2.6 \pm 4.4$ & 15 \\
10 & 25 & 9 & $2.1 \pm 3.2$ & 10 \\
10 & 50 & 4 & $3.5 \pm 4.1$ & 14 \\
10 & 75 & 1 & $7.4 \pm 6.8$ & 24 \\
10 & 100 & 1 & $6.0 \pm 6.6$ & 20 \\
10 & 125 & 4 & $5.0 \pm 5.1$ & 14 \\
10 & 150 & 2 & $9.2 \pm 7.3$ & 28 \\
10 & 175 & 6 & $2.7 \pm 3.3$ & 10 \\
10 & 200 & 5 & $3.5 \pm 4.7$ & 17 \\
10 & 226 & 9 & $3.0 \pm 6.3$ & 24 \\
10 & 249 & 5 & $4.5 \pm 4.7$ & 13 \\
\hline
\end{tabular}

based on primer set $\mathrm{E}+\mathrm{AC} / \mathrm{M}+\mathrm{CTT}$ is shown in Fig. 3. A total of 16 AFLP markers that are present only in 'Afourer' mandarin and not in other citrus were used to study the 'Nules' seedlings from Bakersfield and to determine if 'Afourer' mandarin was the pollen parent (Table 2). Seven 'Minneola' tangelo and three 'Nules' clementine mandarin-specific AFLP markers were used to study the 'Afourer' mandarin seedlings from Madera.

AFLP ANALYSES OF 'NULES' CLEMENTINE SEEDLINGS FROM BAKERSFIELD ORCHARD. Five AFLP primer sets were used to screen the 'Nules' clementine mandarin seedlings from Bakersfield (Table 2). By following a total of 16 AFLP markers that are present only in 'Afourer' mandarin but not in 'Nules' clementine, we were able to identify all 73 'Nules' seedlings tested to have 'Afourer' mandarin as pollen parents. These 73 seedlings derived from the fruit collected from trees $25,100,150,175$, and 249 of row 10; tree 10 of row 30; tree 11 of row 60 ; and tree 10 of row 75 . Fifteen of the 16 'Afourer' mandarin-specific markers segregated in the seedlings and one marker was present in all the seedlings. Seven AFLP markers present in the 'Nules' clementine but not in 'Afourer' mandarin were all segregated in the seedlings. AFLP bands that are present only in navel orange or Valencia orange were not found in any of the 'Nules' seedlings tested.

AFLP ANALYSES OF 'AFOURER' MANDARIN SEEDLINGS FROM MADERA ORCHARD. Ten AFLP primer sets were used to determine the pollen parentage of 'Afourer' mandarin seedlings from the Madera orchard, including seven 'Minneola' tangelo-specific AFLP bands and three clementine mandarin-specific AFLP bands (Table 2). All 10 markers were heterozygous either in the 'Minneola' tangelo or clementine mandarins and were segregated among the 'Afourer' mandarin seedlings. Among 114 'Afourer' mandarin seedlings from the fruit collected at the west end of the 'Afourer' mandarin block, 30 seedlings were identified to have clementine mandarin-specific AFLP bands. Among the 158 'Afourer' mandarin seedlings from the fruit collected at the east end of the 'Afourer' mandarin block, 21 seedlings were identified to contain clementine mandarin-specific AFLP bands and 11 seedlings were found to possess 'Minneola' tangelo-specific AFLP bands. There were no 'Owari' satsuma mandarin or 'Lane Late' navel orange-specific AFLP bands present in the 'Afourer' mandarin seedlings.

\section{Discussion}

RELATIONSHIP BETWEEN FRUIT SIZE, FRUIT WEIGHT, AND SEED NUMBER OF 'Nules' Clementine AND 'AfOURER' MANDARIN. Clementine mandarins have self-incompatibility systems, however, cross-pollination with other citrus is known to enhance fruit set, yield, fruit size, and seed content (De Lange et al., 1979; Eti and Stosser, 1992; Garcia-Papi and Garcia-Martinez, 1984). Lack of cross-pollination can result in low fruit set and requires the use of gibberellic acid to ensure proper fruit set in clementine mandarin production (Ben-Cheikh et al., 1997; Del Rivero et al., 1969; El-Otmani et al., 1992, 2000; Fornes et al., 1992). There was a positive relationship between the seed content and fruit size of clementine mandarins and 'Afourer' mandarin. Fruit with seeds tend to have larger size fruit (Chao, 2005). Fruit size of 'Afourer' mandarin has been used as an indirect grading criterion in the packinghouse to minimize the seed content of fruit.

AFLP MARKERS FOR POLLEN PARENTAGE IDENTIFICATION. In the current study, AFLP markers proved to be a very useful system. The AFLP markers we selected in this study had bands present only in the potential pollen parents, not in the female parents. 


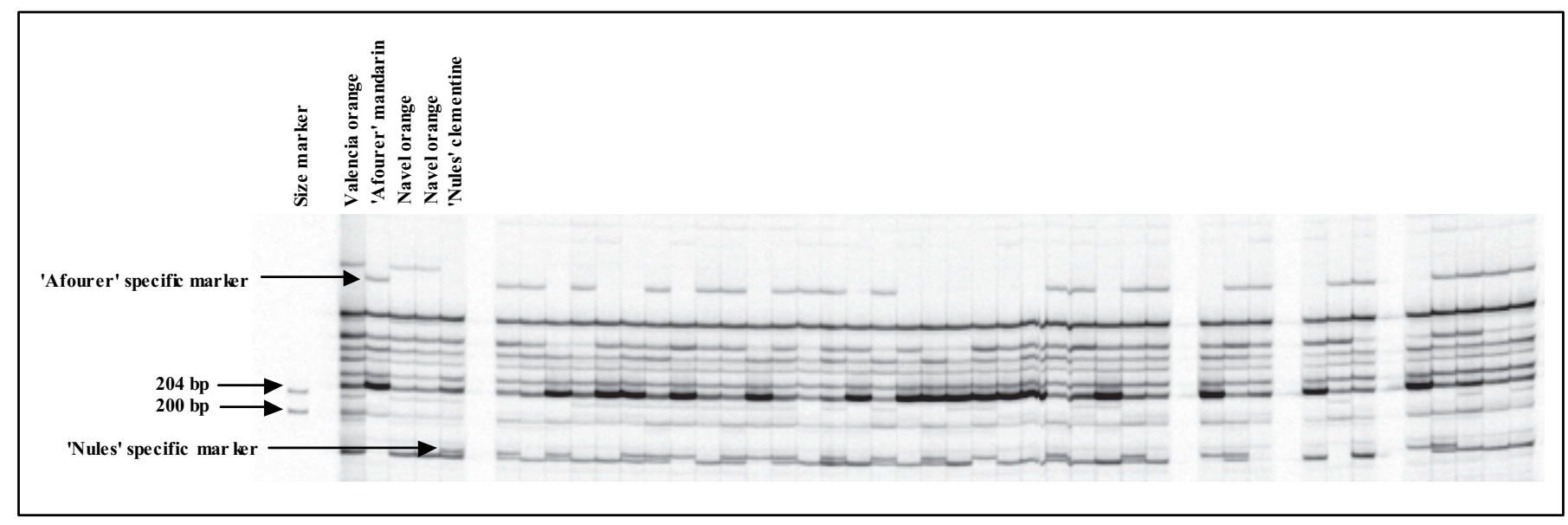

Fig. 3. Molecular size markers and AFLP profiles of parental citrus cultivars and 38 'Nules' clementine seedlings from an orchard near Bakersfield, Calif., generated by using AFLP primer set EcoR I+AC/Mse I+CTT.

Table 2. Orchard location [Bakersfield, Calif. (BAK) or Madera, Calif.], the AFLP primer set combination (EcoR I +2 bases / Mse I + 3 bases), number of cultivar-specific markers of each primer set, and the number of heterozygous or homozygous AFLP markers identified in the pollen parentage study of mandarin seedlings.

\begin{tabular}{lccccc}
\hline Location & Primer sets & $\begin{array}{c}\text { Afourer markers } \\
\text { (no.) }\end{array}$ & $\begin{array}{c}\text { Heterozygous/homozygous } \\
\text { markers (no.) }\end{array}$ & $\begin{array}{c}\text { Nules } \\
\text { markers (no.) }\end{array}$ & $\begin{array}{c}\text { Heterozygous/homozygous } \\
\text { markers (no.) }\end{array}$ \\
\hline BAK & E+TA/M+CTC & 4 & $4 / 0$ & 3 & $3 / 0$ \\
BAK & E+AC/M+CTC & 3 & $2 / 1$ & 0 & $0 / 0$ \\
BAK & E+TA/M+CTT & 4 & $4 / 0$ & 2 & $2 / 0$ \\
BAK & E+AC/M+CTT & 2 & $2 / 0$ & 2 & $2 / 0$ \\
BAK & E+TG/M+CTG & 3 & $3 / 0$ & 0 & $0 / 0$
\end{tabular}

\begin{tabular}{lccccc}
\hline Location & Primer sets & $\begin{array}{c}\text { Minneola markers } \\
\text { (no.) }\end{array}$ & $\begin{array}{c}\text { Heterozygous/homozygous } \\
\text { markers (no.) }\end{array}$ & $\begin{array}{c}\text { Clementine } \\
\text { markers (no.) }\end{array}$ & $\begin{array}{c}\text { Heterozygous/homozygous } \\
\text { markers (no.) }\end{array}$ \\
\hline Madera & E+TG/M+CTG & 3 & $3 / 0$ & 1 & $1 / 0$ \\
Madera & E+AT/M+CTG & 0 & $0 / 0$ & 2 & $2 / 0$ \\
Madera & E+TA/M+CTC & 2 & $2 / 0$ & 0 & $0 / 0$ \\
Madera & E+AC/M+CTC & 2 & $2 / 0$ & 0 & $0 / 0$ \\
\hline
\end{tabular}

Screening through only 32 AFLP primer sets, we were able to identify multiple cultivar-specific markers that were present only in that particular cultivar and absent in all other citrus tested. Among the 23 cultivar-specific AFLP markers we selected for the study, 22 of them were heterozygous in the parents and were segregated among the seedlings. Only one 'Afourer' mandarinspecific marker used in the study of seedlings from Bakersfield was homozygous in 'Afourer' mandarin and all seedlings had that specific AFLP band. Because most citrus, including mandarins, have hybrid origins (Moore, 2001), it is not surprising that the majority of the AFLP markers examined in this study were heterozygous in the pollen parents.

In the marker screening, several AFLP bands that were present only in the navel oranges or 'Owari' satsuma mandarin and not in other mandarins were also identified. These bands were navel orange-specific markers or 'Owari' satsuma mandarin-specific markers. None of these navel oranges or 'Owari' satsuma mandarin-specific markers showed up in any of the seedlings tested from either orchard. Although, both navel oranges and 'Owari' satsuma mandarin are known to have sterile pollen, the absence of any navel oranges or 'Owari' satsuma mandarin-specific markers in the seedlings was additional proof that no pollen from either one caused any seeds in 'Nules' clementine mandarin or 'Afourer' mandarin.
Pollen parentage determination of 'Nules' Clementine SEEDLINGS From BAKERSFIELD. All 73 'Nules' clementine seedlings from the Bakersfield orchard were progenies of 'Afourer' mandarin. Pollen of 'Afourer' mandarin was able to disperse across a minimum of 91 rows of navel oranges (a distance of 570 $\mathrm{m}$, from the edge of the 'Afourer' mandarin block in the north to the edge of the 'Nules' clementine block) from the north or 29.9 ha of empty ground (a distance of $437 \mathrm{~m}$, from the edge of the 'Afourer' mandarin block in the east to the edge of the 'Nules' clementine block) from the east to pollinate the 'Nules' clementine mandarins at the southwest corner of the orchard (Fig. 1). Tree 10 of row 75 was the furthest 'Nules' tree from the potential 'Afourer' mandarin pollen sources. If we consider the distance of this tree to the potential 'Afourer' mandarin pollen source, pollen of 'Afourer' mandarin was able to disperse a minimum distance of $960 \mathrm{~m}$ from the north or a minimum of $837 \mathrm{~m}$ from the east to pollinate this particular 'Nules' clementine mandarin. The actual pollen dispersal distance could be even larger, since the pollen of 'Afourer' mandarin could come from anywhere within the 'Afourer' mandarin block and not just from the edge of the block.

Pollen Parentage determination of 'Afourer' mandarin SEEDLINGS FROM MADERA. The study found that $6.9 \%$ (11 out of 158) of the 'Afourer' mandarin seedlings from the east end of 
the 'Afourer' mandarin block had 'Minneola' tangelo as pollen parent. The pollen of 'Minneola' tangelo was able to disperse across a minimum of 92 rows of 'Lane Late' navel orange plus two rows of 'Afourer' mandarin, a minimum distance of $521 \mathrm{~m}$ (Fig. 2). We found $13.3 \%$ (21 out of 158) of the 'Afourer' mandarin seedlings from the east end of the 'Afourer' mandarin block were progenies of 'Afourer' mandarin $\times$ clementine mandarin. The pollen of clementine mandarins was able to disperse across a minimum of 30 rows of 'Owari' satsuma mandarin plus 24 rows of 'Afourer' mandarins to pollinate the 'Afourer' mandarins, a minimum distance of $301 \mathrm{~m}$. We also found $26.3 \%$ (30 out of 114) of 'Afourer' mandarin seedlings from the west end of the 'Afourer' mandarin block had clementine mandarin as pollen parent. The pollen of clementine mandarin was able to disperse across a minimum of 30 rows of 'Owari' satsuma mandarins plus two rows of 'Afourer' mandarins to pollinate the 'Afourer' mandarins, a minimum distance of $181 \mathrm{~m}$. The dispersal distances found in this study for 'Minneola' tangelo pollen or clementine mandarins' pollen are minimum estimations because the 'Minneola' tangelo pollen or clementine mandarin pollen could come from anywhere within the 'Minneola' tangelo block or clementine mandarin block and not just from the edges of either block.

The percentage of 'Afourer' mandarin seedlings that were from cross-pollination was an underestimation. One main reason for the underestimation is that the 'Afourer' mandarin is highly polyembryonic. The majority of seeds of 'Afourer' mandarin have multiple embryos within each seed. It is common to have average two to three embryos from one seed, and sometimes up to five embryos (Chao, unpublished). Some seedlings we tested were apparently nucellar seedlings instead of zygotic seedlings; therefore, the real outcrossing rate could be higher.

IMPLICATION FOR SEEDLESS MANDARIN PRODUCTION. Citrus pollen is not adapted for wind dispersal, and cross-pollination of citrus is accomplished by insects (Free, 1993). Citrus flowers could be visited by different type of insects, but honeybees were the dominant pollinators for citrus (Hassanein and Ibrahim, 1959; Moffett and Rodney, 1971). Both clementine mandarins and 'Afourer' mandarin are self-incompatible and produce seedless fruit if grown in isolation. Fruit set of clementine mandarin could be significantly increased with the addition of a compatible pollen source and honeybee colonies in caged trees (Van Horn and Todd, 1954). 'Afourer' mandarin is a very strong pollinizer and can cause a large number of seeds in 'Nules' and 'Fina Sodea' clementine mandarins. Clementine mandarins cause less seeds in 'Afourer' mandarin when used as pollinizers in hand crosspollination.

Before this study was conducted in California, 5,10 , or 20 rows (30, 61, and $122 \mathrm{~m}$, respectively) of trees such as navel orange or satsuma mandarin were suggested to be an adequate buffer in preventing cross-pollination by honeybees between compatible mandarin cultivars such as 'Afourer' mandarin and clementine mandarins. We did not expect pollen of clementine mandarins or 'Minneola' tangelo able to disperse at such a long distance to pollinate 'Afourer' mandarin in the orchard near Madera. Therefore, we only sampled trees from the east and west edges of the 'Afourer' mandarin block. If we had collected samples across the 'Afourer' mandarin block, we might have learned even more about how far pollen could disperse in this orchard.

In this study, the longest distance we found that compatible pollen was able to disperse was between $837 \mathrm{~m}$ to $960 \mathrm{~m}$ at the Bakersfield orchard. This was much larger than the $50 \mathrm{~m}$ distance reported previously in Australia. Wallace et al. (2002) used iso- zyme markers to determine the effective pollen transfer distance between the pollinizer cultivar 'Ellendale' mandarin and 'Imperial' mandarin in Australia. They found that the 'Ellendale' pollen was able to transfer across more than 10 rows of trees $(>50 \mathrm{~m})$, but the effective pollen transfer distance decreased significantly after three rows of trees $(15 \mathrm{~m})$. The maximum pollen dispersal distance for particular compatible pollen sources at a particular location is difficult to predict. Many factors could affect the behavior of honeybees (Delaplane and Mayer, 2000; Free, 1993) that, in turn, affect the distance, direction, and frequency of the foraging honeybees and the potential dispersal distance of citrus pollen flow. The density of hives, abundance or scarcity of alternative food sources, or differential honeybee preferences for different types of citrus (Moffett et al., 1974) could all affect the dispersal distance of compatible pollen flow in mandarin orchards.

Comparing the pollen dispersal distance from the studies at two orchards, the results suggest that buffer rows, either navel oranges or satsuma mandarins as the case in the orchard near Madera, might limit the movement of honeybees and reduce the pollen dispersal distance. Empty spacing between compatible mandarin cultivars such as the case in the orchard near Bakersfield did not limit the movement of honeybees. The maximum pollen dispersal distance was much larger in the orchard near Bakersfield. All $100 \%$ of 'Nules' clementine seedlings tested were progenies from outcrosses with 'Afourer' mandarin. Only $6.9 \%$ of the 'Afourer' mandarin seedlings from the east end of the block had pollen from 'Minneola' tangelo at Madera. Although, the two orchards are not directly comparable, large blocks of buffer trees such as navel oranges or satsuma mandarins indeed could reduce crosspollination. Another possibility that the cross-pollination rate at Madera site was much lower might due to the lower compatibilities between 'Afourer' mandarin and 'Minneola' tangelo and between 'Afourer' mandarin and clementines. A larger number of buffer rows more than 94, may be up to 116 rows (92 rows of navel orange plus 24 rows of 'Afourer' mandarin) (from the edge of the 'Minneola' block to the west end of the 'Afourer' mandarin block), are needed to prevent cross-pollination by honeybees in the Madera orchard completely. Growers in California, as well in other places, need to consider a large distance of isolation, up to several km or more than 116 rows of buffer trees, to prevent crosspollination by honeybees. Prevention or reduction of honeybee movement in orchards would reduce the frequency of cross-pollination among compatible mandarin cultivars, but this is very difficult to achieve in the multi-faceted agricultural environment in California. Growers need to carefully select the planting site, and pay special attention to potential compatible pollen sources and the distance between them, to ensure the production of completely seedless clementine or 'Afourer' mandarins.

\section{Literature Cited}

Barry, G.H. 1995. A prediction model to determine the cross-pollination ability of Citrus spp. MS Thesis, Univ. of Natal, Pieteermaritzburg, Republic of South Africa.

Ben-Cheikh, W., J. Perez-Botella, F.R. Tadeo, M. Talon, and E. PrimoMillo. 1997. Pollination increases Gibberellin levels in developing ovaries of seeded varieties of citrus. Plant Physiol. 114:557-564.

Bretó, M.P., C. Ruiz, J.A. Pina and M.J. Asíns. 2001. The diversification of Citrus clementina Hort. Ex Tan., a vegetatively propagated crop species. Mol. Phylogenetics Evol. 21:285-293.

Chao, C.T. 2005. Pollination study of mandarins and the effect on seediness and fruit size-Implications for seedless mandarin production. HortScience (In press). 
Chen, J., P.S. Devanand, D.J. Norman, R.J. Henny, and C.T. Chao. 2004. Analysis of genetic relatedness of Dieffenbachia cultivars using AFLP markers. J. Amer. Soc. Hort. Sci. 129:81-87.

De Lange, J.H. and A.P. Vincent. 1979. Pollination requirements of clementine. Citrus \& Subtropical Fruit J. 547:9-14.

De Lange, J.H., A.P. Vincent, W.J. Bruwer, L.A. Von Broembsen, and A.T.E. Lee. 1979. Pollination and girdling studies on clementine. Citrus \& Subtropical Fruit J. 550:6-10.

Delaplane, K.S. and D.F. Mayer. 2000. Crop pollination by bees. CABI Publishing, New York.

Del Rivero, J.M., P. Veyrat, and D. Gomez de Barreda. 1969. Improving fruit set in Clementine mandarin with chemical treatments in Spain. Proc. Intl. Soc. Citriculture 3:1121-1124.

El-Otmani, M., M.C. Ben Ismail, A. Ait Oubahou, and M. Achouri. 1992. Growth regulators use on clementine mandarin to improve fruit set. Proc. Intl. Soc. Citriculture 505-508.

El-Otmani, M., C.W. Coggins, M. Agusti, and C.J. Lovatt. 2000. Plant growth regulators in citriculture: World current uses. Critical Rev. Plant Sci. 19:395-447.

Eti, S. and R. Stosser. 1992. Pollen tube growth and development of ovules in relationship to fruit set in mandarins cv. 'Clementine' (Citrus reticulata Blanco). Acta Hort. 321:621-625.

Fornes, F., P.J.J. van Rensburg, M. Sanchez-Perales, and J.L. Guardiola. 1992. Fruit setting treatments' effect on two clementine mandarin cultivars. Proc. Intl. Soc. Citriculture 489-492.
Free, J.B. 1993. Insect pollination of crops. 2nd ed. Academic, London. Garcia-Papi, M.A. and J.L. Garcia-Martinez. 1984. Fruit set and development in seeded and seedless clementine mandarin. Scientia Hort. 22:113-119.

Hassanein, M.H. and M.M. Ibrahim. 1959. Studies on the importance of insects, especially the honeybee, in pollination of citrus in Egypt. Agr. Res. Rev., Cairo. 37:390-409.

Moffett, J.O. and D.R. Rodney. 1971. Honey bee visits to citrus flowers. J. Arizona Acad. Sci. 6:254-259.

Moffett, J.O., D.R. Rodney, and C.W. Shipman. 1974. Consistency of honeybee visits to flowering citrus trees. Amer. Bee J. 114:21-23.

Moore, G.A. 2001. Oranges and lemons: clues to the taxonomy of Citrus from molecular markers. Trends in Genetics 17:536-540.

Mueller, U.G. and L.L. Wolfenbarger. 1999. AFLP genotyping and fingerprinting. Trends in Ecol. \& Evolution 14:389-394.

Oppenheimer, H.R. 1948. Experiments with unfruitful 'clementine' mandarins in Palestine. Bul. Agr. Res. Sta. Rehovot. 48.

Soost, R.K. 1956. Unfruitfulness in the clementine mandarin. Proc. Amer. Soc. Hort. Sci. 67:171-175.

Soost, R.K. 1969. The incompatibility gene system in Citrus. Proc. First Intl. Citrus Symp. 1:189-190.

Van Horn, C.W. and F.E. Todd. 1954. Bees, bouquets and better tangerines. Progr. Agr. Ariz. 6:11.

Wallace, H.M., B.J. King, and L.S. Lee. 2002. Pollen flow and the effect on fruit size in an 'Imperial' mandarin orchard. HortScience 37:84-86. 